\title{
Safety window for the transsphenoidal approach for pituitary tumours: a computed tomographic angiography study
}

\author{
F.J. Arrambide-Garza1*(D) P.P. Zarate-Garza ${ }^{1 *(1)}$, K. Aguilar-Morales $^{1}(\mathbb{1}$, \\ I.S. Villarreal-del-Bosque ${ }^{(1)}$, A. Quiroga-Garza ${ }^{1}{ }^{(0)}$, A. Gómez-Sánchez ${ }^{1}{ }^{\circledR}$,

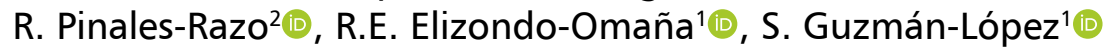 \\ ${ }^{1}$ Universidad Autónoma de Nuevo León, School of Medicine, Human Anatomy Department, \\ Monterrey, Nuevo León, México \\ ¿Universidad Autónoma de Nuevo León, University Hospital "Dr. José Eleuterio González", \\ Radiology and Imaging Department, Monterrey, Nuevo León, México
}

[Received: 7 October 2021; Accepted: 5 November 2021; Early publication date: 16 November 2021]

Background: The aims are to evaluate the morphometry of the sellar region and propose a safety window on the floor of the sella turcica for the transsphenoidal approach in a Hispanic population.

Materials and methods: We retrospectively analysed 150 computed tomographic angiography sellar region images from asymptomatic patients. The images were evaluated intraobservatory by an expert radiologist. We measured: intercarotid distance of cavernous segment; depth of sella turcica; skull base angle; anterior distance, the distance between anterior spinal nasal and floor of the sella turcica; posterior distance, the distance between anterior spinal nasal and posterior wall of the sella turcica; anterior surgical angle, formed between the floor of the nostril and superior limit of the anterior wall of the sella turcica; and posterior angle, formed between the floor of the nostril and the inferior limit of the posterior wall of the sella turcica.

Results: Safety window was based on two measures: the intercarotid distance and depth. The mean of the safety window is $151.13 \mathrm{~mm}^{2}$ and $147.60 \mathrm{~mm}^{2}$ for men and women, respectively. The intercarotid distance was $17.83 \mathrm{~mm}$. The depth of the sella turcica was $8.46 \mathrm{~mm}$. The skull base angle was 112.13 grades. The anterior distance was $76.34 \mathrm{~mm}$. The posterior distance was $87.59 \mathrm{~mm}$. The anterior surgical angle was 32.76 grades. The posterior surgical angle was 87.59 grades. Conclusions: The surgical approach space is smaller in females. It could significate a more complicated surgery in this population. Anatomical understanding could reduce complications in hospitals without a neuronavigation system. (Folia Morphol 2023; 82, 1: 17-23)

Key words: computed tomographic, internal carotid artery, safety window, sella turcica, transsphenoidal surgery

Address for correspondence: S. Guzmán-López, MD, PhD or R.E. Elizondo-Omaña, MD, PhD, Human Anatomy Department, Faculty of Medicine and University Hospital "Dr. José Eleuterio González", Universidad Autónoma de Nuevo León (UANL), Monterrey, Nuevo León, México, tel: +52 (81)83-29-41-71, e-mails: dr.santos.anato@gmail.com or rod_omana@yahoo.com

*Both authors participated equally in the study, and both should be considered in the position of first author.

This article is available in open access under Creative Common Attribution-Non-Commercial-No Derivatives 4.0 International (CC BY-NC-ND 4.0) license, allowing to download articles and share them with others as long as they credit the authors and the publisher, but without permission to change them in any way or use them commercially. 


\section{INTRODUCTION}

The pituitary gland is a structure located in the middle cranial fossa, inside in the sella turcica $[14,15]$. It is conformed by the tuberculum sellae, the hypophyseal fossa, and the dorsum sellae $[10,24]$. Is reported that $19 \%$ of central nervous system tumours originate in the sellar region and pituitary adenomas are the most common tumours in this region [3]. The general prevalence of pituitary adenomas is $16.7 \%$ [11]. These tumours are classified as microadenomas when their size is less than $10 \mathrm{~mm}$, and as macroadenomas when their size is greater than $10 \mathrm{~mm}[12,19,20]$. Treatment options include endoscopic endonasal transsphenoidal surgery (EETS), which is reserved mainly for patients whose symptoms are associated with tumour size, tumour invasion to adjacent structures, and tumours that do not respond to drug treatment $[8,23]$.

Endoscopic endonasal transsphenoidal surgery is divided into nasal, sphenoidal, and sellar phases. The opening of the sellar floor and the resection of the tumour is performed in the sellar phase. It is a challenging procedure due to the complexity of the anatomical region and the limited surgical area $[1,5]$. Common complications include intercavernous sinus bleeding and internal carotid artery (ICA) laceration $[6,9,13]$. Part of the ICA is located inside the cavernous venous sinus until it passes through the dura mater that forms the roof of the venous sinus $[7,28]$. A thorough understanding of the nasal cavity and middle cranial fossa is needed to improve patient safety during these procedures. This study aims to evaluate the morphometry of the region and propose a safety window on the floor of the sella turcica.

\section{MATERIALS AND METHODS}

An observational, cross-sectional, retrospective and descriptive study was performed. Computed tomographic angiography (CTA) studies were obtained from the database of the Radiology and Imaging Department of the University Hospital between April 2015 and July 2018. Sampling was done in consecutive cases. Studies from Hispanic patients from northeast of México with an age between 18 and 86 who underwent a head and neck CTA indicated by their treating physician. We categorised the population into groups according to their sex. Those patients with sellar or parasellar disease, central nervous system vascular disease, previous cranial surgery, or other central nervous system diseases that may alter anatomy, were excluded. Studies with abnormalities or artefacts were eliminated.

\section{Study technique}

We used a 64-slice computed tomography (General Electric CT99 Light Speed VCT), Software $12 \mathrm{HW} 14.6$, using the following parameters: rotation of $0.4 \mathrm{~s}$ helical acquisition, coverage of detectors of $20 \mathrm{~mm}, 120 \mathrm{kV}, 400$ more, cutting thickness of $0.625 \mathrm{~mm}$, Pitch of $0.53: 1 \mathrm{~mm} / \mathrm{rot}$, and field o view from 22 to $23 \mathrm{~cm}$. All the imaging data was uploaded to the Carestream Vue PACS and analysed in coronal and sagittal planes for the following parameters (Fig. 1):

- intercarotid distance: the smallest distance between medial walls of the cavernous segment of ICA;

- depth of sella turcica: the greatest distance between the floor and a perpendicular line connecting the tuberculum and dorsum;

- skull base angle: the angle formed between the planum clival and the clivus;

- anterior surgical angle: the angle formed between the floor of the nostril and the superior limit of the anterior wall of the sella turcica;

- posterior surgical angle: the angle formed between the floor of the nostril and the inferior limit of the posterior wall of the sella turcica;

- anterior distance: the distance between the anterior nasal spine and the midpoint of the anterior wall of the sella turcica;

- posterior distance: the distance between the anterior nasal spine and the superior border of the posterior wall of the sella turcica;

- safety window of the sella turcica: an area based on intercarotid distance and depth of sella turcica. All parameters were measured twice by a head and neck expert radiologist using a 5 stage zoom and recorded in a database using millimetres with two decimal unit precision. We considered measurements of the intercarotid distance of cavernous segment, skull base angle, and depth of sella turcica from other published papers. All authors agreed to use bone and vascular landmarks to determine the safety window, such as sphenoid bone and ICA. We used a sagittal and coronal plane for all measurements. The first sagittal plane was aligned with the anterior nasal spine, to include all structures within the same plane. The coronal plane was used to measure the smallest distance between the medial walls of the cavernous 


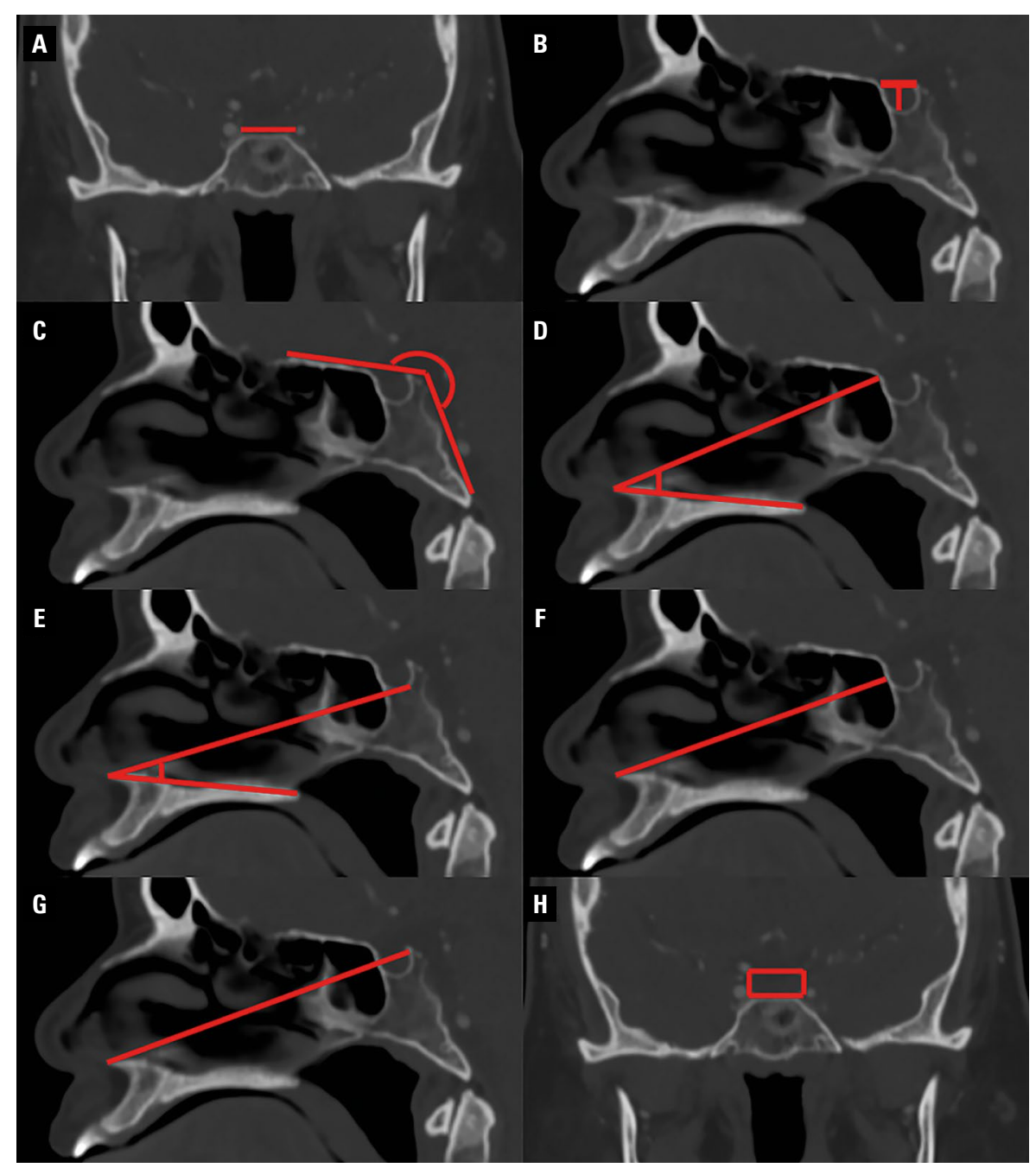

Figure 1. Parameters analysed in coronal and sagittal planes in the sellar region; A. Coronal plane in the sellar region. The intercarotid distance is defined as the smallest distance between the inner walls of the cavernous segment of the internal carotid artery; B-G. Sagittal plane aligned with the anterior nasal spine. B. Sagittal plane. Depth of sella turcica: the greatest distance between the floor and a perpendicular line connecting the tuberculum and dorsum; C. Sagittal plane. Skull base angle: the angle formed between the planum clival and the clivus; D. Sagittal

plane. Anterior surgical angle: the angle formed between the floor of the nostril and upper limit of the anterior wall of the sella turcica; $\mathbf{E}$. Sagittal plane. Posterior surgical angle: the angle formed between the floor of the nostril and the lower limit of the posterior wall of the sella turcica; $\mathbf{F}$. Shows the anterior surgical distance: the distance between the anterior nasal spine and the anterior wall of the sella turcica; $\mathbf{G}$. Sagittal plane. Posterior surgical distance: the distance between the anterior nasal spine and the upper border of the posterior wall of the sella turcica; H. Coronal plane. Safety window on the floor of the sella turcica.

segment of ICA. Once the planes were established, the measurements were manually plotted and adjusted for accuracy by an expert radiologist. All variables were measured, and the process blinded when repeated in the same patient to establish satisfactory intraobserver variability $(\kappa>0.80)$.

\section{Statistical analysis}

The database was analysed using the SPSS Version 24.0 programme for Windows 10 (IBM, Armonk, NY, USA). The normality test was performed using the Kolmogorov-Smirnov test. The mean and standard deviation for each parameter was determined independently. A t Student test was used to determine the statistical significance for the parametric data between men vs. women, and Pearson's $\mathrm{p}$ correlation test for age and each variable. Mann-Whitney $U$ and Kruskal-Wallis were performed for the nonparametric data. The $p$-value of 0.05 was set for statistical significance. Subsequent statistical tests were performed on age subcategorisations or classification by skull base angle categorisation using ANOVA test with post hock adjusted with Tukey and Bonferroni. 
Table 1. Measurements for both sexes

\begin{tabular}{|c|c|c|c|c|}
\hline Measurement & General $(n=150)$ & Males (n = 77) & Females $(\mathrm{n}=73)$ & P-value \\
\hline Intercarotid distance [mm] & $17.83 \pm 4.28$ & $18.81 \pm 4.29$ & $16.75 \pm 4.11$ & $0.003^{*}$ \\
\hline Depth of sella turcica [mm] & $8.46 \pm 1.34$ & $8.05 \pm 1.19$ & $8.85 \pm 1.44$ & $<0.001^{*}$ \\
\hline Anterior angle approach $\left[^{\circ}\right]$ & $32.76 \pm 2.95$ & $32.47 \pm 2.80$ & $33.04 \pm 3.20$ & 0.244 \\
\hline Posterior angle approach [ $\left.{ }^{\circ}\right]$ & $25.13 \pm 3.09$ & $25.40 \pm 2.83$ & $24.84 \pm 3.36$ & 0.265 \\
\hline Skull base angle $\left[^{\circ}\right]$ & $112.13 \pm 7.68$ & $111.74 \pm 7.18$ & $112.58 \pm 8.19$ & 0.507 \\
\hline Anterior distance [mm] & $76.34 \pm 5.27$ & $79.52 \pm 4.52$ & $72.99 \pm 3.82$ & $<0.001^{*}$ \\
\hline Posterior distance [mm] & $87.59 \pm 5.21$ & $90.43 \pm 4.97$ & $84.55 \pm 3.48$ & $<0.001^{*}$ \\
\hline Area $\left[\mathrm{mm}^{2}\right]$ & $149.36 \pm 38.90$ & $151.13 \pm 36.96$ & $147.60 \pm 41.06$ & 0.581 \\
\hline
\end{tabular}

Data are shown as mean \pm standard deviation. *Statistical significance

\section{Ethical considerations}

This study was previously reviewed and approved by the University's Ethics and Research Committees with the registration number $\mathrm{AH} 19-00002$ on April 25, 2019, making sure it adheres to the Helsinki declaration and national and international standards of research. The authors declare no financial or commercial gain for the realisation of this study. Also, the authors declare no conflict of interest. None of the imaging studies were performed for the purposes of this study.

\section{RESULTS}

We retrospectively reviewed 150 head and neck CTA with a mean age of $53.51 \pm 15.9$ (range 18-86 years). The sample was stratified by gender with 77 (51.3\%) men with a mean age of $53.21 \pm 15.2$ years, and $73(48.7 \%)$ women with a mean age of $53.85 \pm 16.69$.

Descriptive statistics are shown in Table 1. Significant differences between sexes were found in the intercarotid distance, depth, and the anterior and posterior distances. All distances were smaller in women, except for the depth.

Statistical tests were performed for comparison between subcategorised age groups divided into ranks by decades however no statistical significance was found among the groups. Correlation tests were performed by Pearson correlation coefficient finding the following values: intercarotid distance -0.136 , the height of the sella turcica 0.122 , anterior surgical angle 0.127 , posterior surgical angle 0.089 , skull base angle -0.140 , anterior distance -0.52 , posterior distance -0.004 , and area -0.028 .

\section{Skull base angle}

Subcategorisation was performed at the skull base angle. These were divided into horizontal (> $\left.121^{\circ}\right)$ with $24(15.6)$ patients, normal (120-105') with $106(70.6 \%)$ patients, and vertical $\left(<104^{\circ}\right)$ with $20(13.3 \%)$ patients without statistical difference between them.

\section{DISCUSSION}

Endoscopic endonasal transsphenoidal surgery has been developed in the last 10 years, currently improving outcomes due to a better understanding of the local anatomy, and technological and surgical advancement. A clear example is the neuronavigation system. It consists of a set of computer-assisted technologies to guide the surgeon within the skull, providing the best surgical planning/safety, and decreasing trans-operating complications. The use of this system is recommended in all neurosurgical approaches. The image-guided neurosurgery is used to perform tumour resection, treatment of vascular disease, epilepsy surgery, and biopsies. During the surgery, the neuronavigation system permits a precise localisation of the region of interest and it also allows the visualisation of the instrument movements inside the skull. It helps to determine the difference between the healthy brain and the pathologic process that could be undifferentiated in a macroscopic view [29]. Although the neuronavigation improves the neurosurgical performance, anatomical knowledge is still essential for a precise surgery.

Intercarotid distance and depth of the sella turcica determines a corridor for the EETS and others skull base surgeries. Different methods have been used for estimate it, these variables may be measured using CTA as a reproducible method. Our findings show differences between sexes in the intercarotid distance, depth, anterior and posterior distance. This data suggests a smaller space for women during the surgical approach. Moreover, all these findings are of importance due to differences with other pop- 
Table 2. Comparative with other studies

\begin{tabular}{|c|c|c|c|c|}
\hline Measurement & Arrambide-Garza et al. (2020) & Nunes et al. (2015) & Mascarella et al. (2015) & Shrestha et al. (2018) \\
\hline Country & México & Brazil & Canada & Nepal \\
\hline Imaging study & CTA & MRI & CTA & Radiograph \\
\hline Sample size & 150 & 20 & 34 & 40 \\
\hline Intercarotid distance [mm] & $17.83 \pm 4.28$ & $19.41 \pm 3.00$ & $17.3 \pm 0.8$ & ND \\
\hline Depth [mm] & $8.46 \pm 1.34$ & ND & ND & $6.40 \pm 0.92$ \\
\hline Anterior angle approach $\left[^{\circ}\right]$ & $32.76 \pm 2.95$ & ND & ND & ND \\
\hline Posterior angle approach [] & $25.13 \pm 3.09$ & ND & ND & ND \\
\hline Anterior distance [mm] & $76.34 \pm 5.27$ & ND & ND & ND \\
\hline Posterior distance [mm] & $87.59 \pm 5.21$ & ND & ND & ND \\
\hline Area $\left[\mathrm{mm}^{2}\right]$ & $149.36 \pm 38.90$ & ND & ND & ND \\
\hline
\end{tabular}

Data are shown as mean \pm standard deviation. CTA — computed tomographic angiography; MRI — magnetic resonance imaging; ND — not described

Table 3. Classification of planum-clival angle type and comparative with another study

\begin{tabular}{lcc}
\hline Measurement & $\begin{array}{c}\text { Arrambide-Garza } \\
\text { et al. (2020) }\end{array}$ & $\begin{array}{c}\text { Alkherayf et al. } \\
\text { (2015) }\end{array}$ \\
\hline Country & México & Canada \\
Sample size & 150 & 89 \\
Skull base angle type A & $25(15.6 \%)$ & $15(17 \%)$ \\
Skull base angle type B & $106(70.6 \%)$ & $61(69 \%)$ \\
Skull base angle type C & $20(13.3 \%)$ & $13(15 \%)$ \\
\hline
\end{tabular}

ulations (Tables 2, 3). Implementing these findings improve the knowledge of base skull anatomy and could help in the planning of transsphenoidal surgery in those hospitals where a neuronavigation system is not available.

\section{Relevance for the surgical practice}

The sellar region is limited from above by the circle of Willis and chiasm, laterally by cavernous sinuses, and posteriorly by the brainstem, basilar artery, and its branches [25]. EETS can be divided into three phases, nasal, sphenoidal, and sellar, each one with possible complications. In most cases, the surgeon performs the surgery only with anatomical orientation, therefore, we considered the following information is useful to increase the anatomic understanding for each phase.

Nasal and sphenoidal phase. The nasal phase consists of advancing with the endoscope through the floor of the nostril towards the choana; subsequently, the ethmoidal recess is identified, and the posterior portion of the nasal septum is removed. In the sphenoidal phase, the anterior wall of the sphenoidal sinus is removed. Within the sphenoidal sinus, the floor of the sella turcica should be delimited, including the sphenoidal plane, clivus, the protrusions of both carotid arteries, and the prominence of the optic nerves [4].

Landmarks have been reported to orient the surgical intranasal trajectory. Misalignment during the trajectory could increase the length of the procedure that could lead to a more difficult surgical approach [2]. Alkherayf et al. [2] described a classification of the skull base angle that recommends the head position during the EETS that determines a direct surgical intranasal trajectory. The angle was classified into three types: a) $>121^{\circ}$ is related with a horizontally oriented sella, requiring 10 or 20 degrees of neck flexion; b) $105-120^{\circ}$ requiring neutral head position for a standard sphenoidectomy; and c) $<104^{\circ}$ associated with a vertical oriented sella, this type requires 10 or 20 degrees of neck extension. The skull base angle (Fig. 1C) in our study reveals that type B (105-120') is the most common (70.6\%), requiring neutral head positioning with the standard sphenoidectomy, similar data as Alkherayf et al. [2] in the Canadian population (Table 3 ). This would permit an adequate surgical exposure and a better tumour resection. Although this classification does not supplant the image guidance, it is a practical orientation method for neurosurgeons [2].

The sellar region is limited by a small space. We propose the anterior and posterior surgical angles that correspond to the anterior and posterior limits of the sella turcica in a sagittal plane (Fig. 1D, E) to determine the angle approach in the surgery and limit the space of the sellar region, therefore, the surgeon would not reach the anterior or posterior cranial fossa. The superior limit of the anterior aspect 
of the sella turcica, and the inferior limit of the dorsum sellae, respectively. Our results show an anterior surgical angle of $32.47 \pm 2.80^{\circ}$ and posterior surgical angle of $25.40 \pm 2.83^{\circ}$ for males, with a range of motion of 7.02 degrees. In females, the anterior and posterior angles approach were $33.04 \pm 3.19^{\circ}$ and $24.84 \pm 3.36^{\circ}$, respectively, with a range of motion of 8.2 degrees. We do not obtain statistical differences between sexes.

We also propose the anterior distance which oriented the interval between the sphenoid bone and the anterior nasal spine. We obtain statistical differences in the anterior distance between sexes (Fig. $1 \mathrm{~F}$ ) is $79.52 \mathrm{~mm}$ in males, and $72.99 \mathrm{~mm}$ in females. Exist roughly $10 \mathrm{~mm}$ of difference between sexes to reach the sellar region that suggest a more distance in males and it could help in surgical planning.

Sellar phase. The surgeon opens the sellar floor and the resection of the tumour is performed [4]. The most important complication in this phase is the intrasellar haemorrhage due to a laceration of the ICA, which can potentially result in death [26]. We described a safety window based on two measures: the intercarotid distance and depth.

Lin et al. [16] determined a correlation between the intercarotid distance in the cavernous segment and non-functional pituitary macroadenomas treated with EETS, as these modify the position of non-bone structures. Our findings are from healthy patients, therefore, can be only applied in microadenomas due to the anatomy is respected. We report a mean intercarotid distance (Fig. 1A) in the sellar region of $17.83 \mathrm{~mm}$, similar data as Mascarella et al. [17] who reported a mean of $17.3 \mathrm{~mm}$ using CTA in a Canadian population (Table 2). However, Nunes et al. [21] reported a larger mean of $19.41 \mathrm{~mm}$ in the Brazilian population using enhanced-magnetic resonance imaging (Table 2). These differences could be related to the method used to measure the intercarotid distance and ethnic differences among the population.

We did not obtain a statistical difference between sexes in the depth of the sella turcica but, we measured roughly $1 \mathrm{~cm}$ of difference, in males $8.05 \mathrm{~mm}$ and $8.85 \mathrm{~mm}$ in women. This data determines the range motion in a vertical plane within sella turcica, a higher height in females could be an advantage during EETS. The mean depth of the sella turcica of our study (Fig. 1B) was $8.46 \pm 1.34 \mathrm{~mm}$, larger than the reported $6.40 \pm 1.21$ by Shrestha et al. [26] in the Nepal population. This extra $25 \%$ of depth in our population, allows the surgeon more space for the incision and a possible advantage in the performing of the procedure. These differences between the populations are attributed to genetics and environmental factors.

The safety window on the floor of the sella turcica is $151.13 \pm 36.96 \mathrm{~mm}^{2}$ and $147.60 \pm 41.06 \mathrm{~mm}^{2}$ for males and females, respectively (Fig. 1H). The intercarotid distance has an important role in EETS, it determines the space for the surgery. We obtain statistical differences in the intercarotid distance between sexes, $18.81 \mathrm{~mm}$ in males and $16.75 \mathrm{~mm}$ in females. It is also important to consider gender, as women tend to have a higher prevalence of adenomas and smaller distances and areas, the surgical approach could be more difficult in females than males [18].

We can find the thalamoperforating arteries posterior to the sellar region. These arteries supply irrigation to the midbrain and thalamus $[22,27,30]$ and are a potential complication when the tumour is being removed. To avoid these arteries, we propose an anatomic reference, the posterior distance that is the distance to reach the dorsum sellae from the anterior nasal spine (Fig. 1G). We obtained statistical differences between sexes in the posterior distance, the mean is $90.43 \pm 4.97 \mathrm{~mm}$ in men and $84.55 \pm$ $\pm 3.48 \mathrm{~mm}$ in women.

\section{Strengths and limitations}

Our study provides useful information for a better anatomical understanding of the nasal fossa and middle cranial fossa. Nonetheless, there are some limitations. Some of the measurements have not been validated by other studies, such as anterior and posterior approach angles, safety window, anterior and posterior distances. The measurements obtained by imaging are constant with bone structures, which may not be the case in the patient's anatomy due to soft tissue. The patients' anthropometric characteristics were not included in the analysis. The study population only includes Hispanic patients, therefore, further data is needed to compare results with other populations. Morphometric parameters reported are from normal sellar anatomy. Patients that require a transsphenoidal approach to the sellar region may present distorted anatomy due to large tumours like pituitary macroadenomas, therefore results are most useful to guide surgeons in smaller pathologies, such as pituitary microadenomas. Future studies could analyse differences between patients with sellar and parasellar tumours, to compare with a control group. 


\section{CONCLUSIONS}

The differences in the variables measured between sexes are important due to greater difficulty in the female population because the surgical approach space is smaller and a higher prevalence of pituitary adenomas. Understanding the morphometrical variations of the sella turcica improves the anatomical knowledge for radiologists and neurosurgeons in preoperative planning.

\section{Acknowledgements}

We thank all authors for revision and writing assistance. Further, we thank Alejandro Quiroga Garza for providing language help and guidance in the writing process.

\section{Conflict of interest: None declared}

\section{REFERENCES}

1. Ajler $\mathrm{P}, \mathrm{Hem} \mathrm{S}, \mathrm{Goldschmidt} \mathrm{E}$, et al. [Endoscopic transnasal surgery for pituitary adenomas]. Surg Neurol Int. 2012; 3(Suppl 6): S389-S394, doi: 10.4103/2152-7806.104403, indexed in Pubmed: 23596553.

2. Alkherayf F, Edem I, Ouattara JM, et al. Planum-Clival angle classification: a novel preoperative evaluation for sellar/parasellar surgery. J Neurol Surg B Skull Base. 2015; 76(4): 316-322, doi: 10.1055/s-0035-1549002, indexed in Pubmed: 26225323.

3. Anaya-Delgadillo G, de Juambelz-Cisneros PP, Fernández-Alvarado $B$, et al. [Prevalence of central nervous system tumours and histological identification in the operated patient: 20 years of experience]. Cir Cir. 2016; 84(6): 447-453, doi: 10.1016/j.circir.2016.01.004, indexed in Pubmed: 27094784.

4. Cappabianca P, Cavallo L, de Divitiis E. Endoscopic endonasal transsphenoidal surgery. Neurosurgery. 2004; 55(4): 933-941, doi: 10.1227/01.neu.0000137330.02549.0d.

5. Cavallo LM, Somma T, Solari D, et al. Endoscopic endonasal transsphenoidal surgery: history and evolution. World Neurosurg. 2019; 127: 686-694, doi: 10.1016/j. wneu.2019.03.048, indexed in Pubmed: 31266131.

6. Charalampaki P, Ayyad A, Kockro RA, et al. Surgical complications after endoscopic transsphenoidal pituitary surgery. J Clin Neurosci. 2009; 16(6): 786-789, doi: 10.1016/j. jocn.2008.09.002, indexed in Pubmed: 19289287.

7. Chin OY, Ghosh R, Fang $\mathrm{CH}$, et al. Internal carotid artery injury in endoscopic endonasal surgery: A systematic review. Laryngoscope. 2016; 126(3): 582-590, doi: 10.1002/ lary.25748, indexed in Pubmed: 26525334.

8. Christian E, Harris B, Wrobel B, et al. Endoscopic endonasal transsphenoidal surgery: implementation of an operative and perioperative checklist. Neurosurg Focus. 2014; 37(4): E1, doi: 10.3171/2014.7.FOCUS14360, indexed in Pubmed: 25270128

9. Deng X, Chen S, Bai Ya, et al. Vascular complications of intercavernous sinuses during transsphenoidal surgery: an anatomical analysis based on autopsy and magnetic resonance venography. PLoS One. 2015; 10(12): e0144771, doi: 10.1371/journal. pone.0144771, indexed in Pubmed: 26658152.

10. Di lorgi N, Morana G, Gallizia AL, et al. Pituitary gland imaging and outcome. Endocr Dev. 2012; 23: 16-29, doi: 10.1159/000341736, indexed in Pubmed: 23182817.

11. Ezzat S, Asa SL, Couldwell WT, et al. The prevalence of pituitary adenomas: a systematic review. Cancer. 2004; 101(3): 613-619, doi: 10.1002/cncr.20412, indexed in Pubmed: 15274075.
12. Go JL, Rajamohan AG. Imaging of the Sella and Parasellar Region. Radiol Clin North Am. 2017; 55(1): 83-101, doi: 10.1016/j.rcl.2016.09.002, indexed in Pubmed: 27890190.

13. Hofstetter CP, Shin BJ, Mubita L, et al. Endoscopic endonasal transsphenoidal surgery for functional pituitary adenomas. Neurosurg Focus. 2011; 30(4): E10, doi: 10.3171/2011.1.FOCUS10317, indexed in Pubmed: 21456921.

14. Hong GK, Payne SC, Jane JA. Anatomy, physiology, and laboratory evaluation of the pituitary gland. Otolaryngol Clin North Am. 2016; 49(1): 21-32, doi: 10.1016/j. otc.2015.09.002, indexed in Pubmed: 26614827.

15. Korkmaz M, Yildirim AE, Osun A, et al. Endoscopic endonasal transsphenoidal pituitary surgery: experience of 80 cases. Turk Neurosurg. 2015; 25(6): 883-887, doi: 10.5137/1019-5149.JTN.10495-14.2, indexed in Pubmed: 26617137.

16. Lin BJ, Chung TT, Lin MC, et al. Quantitative analysis of anatomical relationship between cavernous segment internal carotid artery and pituitary macroadenoma. Medicine (Baltimore). 2016; 95(41): e5027, doi: 10.1097/ MD.0000000000005027, indexed in Pubmed: 27741111.

17. Mascarella MA, Forghani R, Di Maio S, et al. Indicators of a reduced intercarotid artery distance in patients undergoing endoscopic transsphenoidal surgery. J Neurol Surg B Skull Base. 2015; 76(3): 195-201, doi: 10.1055/s-00341396601, indexed in Pubmed: 26225301.

18. McDowell BD, Wallace RB, Carnahan RM, et al. Demographic differences in incidence for pituitary adenoma. Pituitary. 2011; 14(1): 23-30, doi: 10.1007/s11102-0100253-4, indexed in Pubmed: 20809113.

19. Mercado M, Melgar V, Salame L, et al. Clinically non-functioning pituitary adenomas: Pathogenic, diagnostic and therapeutic aspects. Endocrinol Diabetes Nutr. 2017; 64(7): 384-395, doi: 10.1016/j.endinu.2017.05.009, indexed in Pubmed: 28745610.

20. Molitch ME. Diagnosis and treatment of pituitary adenomas: a review. JAMA. 2017; 317(5): 516-524, doi: 10.1001/ jama.2016.19699, indexed in Pubmed: 28170483.

21. Nunes CF, Cabral GA, Mello Junior JO, et al. Pituitary macroadenoma: analysis of intercarotid artery distance compared to controls. Arq Neuropsiquiatr. 2016; 74(5): 396-404, doi: 10.1590/0004-282X20160046, indexed in Pubmed: 27191236.

22. Park SQ, Bae HG, Yoon SM, et al. Morphological characteristics of the thalamoperforating arteries. J Korean Neurosurg Soc. 2010; 47(1): 36-41, doi: 10.3340/ jkns.2010.47.1.36, indexed in Pubmed: 20157376.

23. Pinar E, Yuceer N, Imre A, et al. Endoscopic endonasal transsphenoidal surgery for pituitary adenomas. J Craniofac Surg. 2015; 26(1): 201-205, doi: 10.1097/ SCS.0000000000001240, indexed in Pubmed: 25469897.

24. Rajaratnam S. Pituitary gland imaging. Indian J Endocrinol Metab. 2013; 17(Suppl 1): S100-S101, doi: 10.4103/22308210.119516, indexed in Pubmed: 24251122.

25. Rhoton A. The sellar region. Neurosurgery. 2002 ; 51(suppl_4): S1-335-S1-374, doi: 10.1097/0000612320021000̄1-00009.

26. Shrestha GK, Pokharel PR, Gyawali R, et al. The morphology and bridging of the sella turcica in adult orthodontic patients. BMC Oral Health. 2018; 18(1): 45, doi: 10.1186/ s12903-018-0499-1, indexed in Pubmed: 29548316.

27. Uz A. Variations in the origin of the thalamoperforating arteries. J Clin Neurosci. 2007; 14(2): 134-137, doi: 10.1016/j. jocn.2006.01.047, indexed in Pubmed: 17113294.

28. Vijaywargiya M, Deopujari R, Athavale SA. Anatomical study of petrous and cavernous parts of internal carotid artery. Anat Cell Biol. 2017; 50(3): 163-170, doi: 10.5115/ acb.2017.50.3.163, indexed in Pubmed: 29043093.

29. Wang EW, Gardner PA, Zanation AM. International consensus statement on endoscopic skull-base surgery: executive summary. Int Forum Allergy Rhinol. 2019; 9(S3): S127-S144, doi: 10.1002/alr.22327, indexed in Pubmed: 30957956.

30. Weidenbecher M, Huk WJ, Iro H. Internal carotid artery injury during functional endoscopic sinus surgery and its management. Eur Arch Otorhinolaryngol. 2005; 262(8): 640-645, doi: 10.1007/s00405-004-0888-8, indexed in Pubmed: 15657746. 